\title{
Freeze-fracture Electron Microscopy on Microbubbles Used as Theranostics or Made of Lung Surfactant
}

\author{
Brigitte Papahadjopoulos-Sternberg and Jack Ackrell
}

NanoAnalytical Laboratory, San Francisco, California, USA

Microbubble suspensions are frequently used for biomedical applications such as ultrasound contrast enhancement, ultrasound-promoted drug and gene delivery, and blood substitution. The potency of such microbubbles, used as theranostics, is strongly depending upon their coating material and their morphology adopted in a biological relevant environment. Furthermore, the stability of lung surfactant microbubbles, derived from amniotic fluid, is widely used for the prediction of neonatal respiratory distress syndrome.

Freeze-fracture electron microscopy (ff-EM) as a cryofixation replica technique is a powerful tool to visualize microbubbles in a probe-free mode. Since beam-damage resistant replica can be produced from micro-meter size objects, ff-EM allows us to study nano-scale events, such as transient structural inhomogeneities in the bubble coating material, in micro-scale artificial assemblies, such as microbubbles. Since the resolution limit of ff-EM is in our hands $2 \mathrm{~nm}$ for periodical structures we obtained detailed information about the fine structure of bubble coatings. Moreover, the fact that the fracture plane follows the area of weakest forces allows insides into the coating material/gas interface exploring structural inhomogeneities such as domains/rafts in these otherwise inaccessible areas.

We studied the morphology of lipid-, polymer-, and surfactant-stabilized microbubbles by ff-EM.

Freeze-fracture electron micrographs of lipid-stabilized gasbubbles display concave fracture planes only (shadow in front of the structures, Fig. 1). This fracture behavior provides the proof that these microbubbles are coated by a lipid monolayer $[1,2]$. Structural inhomogeneities were detected in a certain lipid monolayer type and identified as lipid domains ( $\varnothing 100-250 \mathrm{~nm}$, step height $\sim 20 \mathrm{~nm}$ ) surrounded by a polymer-rich matrix (Fig. 2) [2, 3].

Cross fractions through polymer-stabilized gas bubbles reveal a shell thickness of $\sim 300 \mathrm{~nm}$ stabilizing microbubbles of about $3 \mu \mathrm{m}$ in diameter. Individual polyvinyl alcohol bundles are detectable in the polymer coating (Fig.3) [2].

Microbubbles stabilized by a common exogenous lung surfactant, Survanta, are less than $10 \mu \mathrm{m}$ in diameter and undulation pattern are detected in their monolayer coatings (Fig. 4). This lung surfactant is capable of routinely stabilizing approximately a billion microbubbles per $\mathrm{mL}$ [4].

\section{References}

[1]. C. Brancewicz et al., J. Disp. Science and Technology 27 (2006) 761.

[2]. B. Papahadjopoulos-Sternberg, Liposomes: Methods in Molecular Biology 606, Humana Press, 2010, 333.

[3]. M. A. Borden et al., Langmuir 22 (2006) 4291.

[4]. S. Sirsi et al. Soft Matter 5 (2009) 4835. 


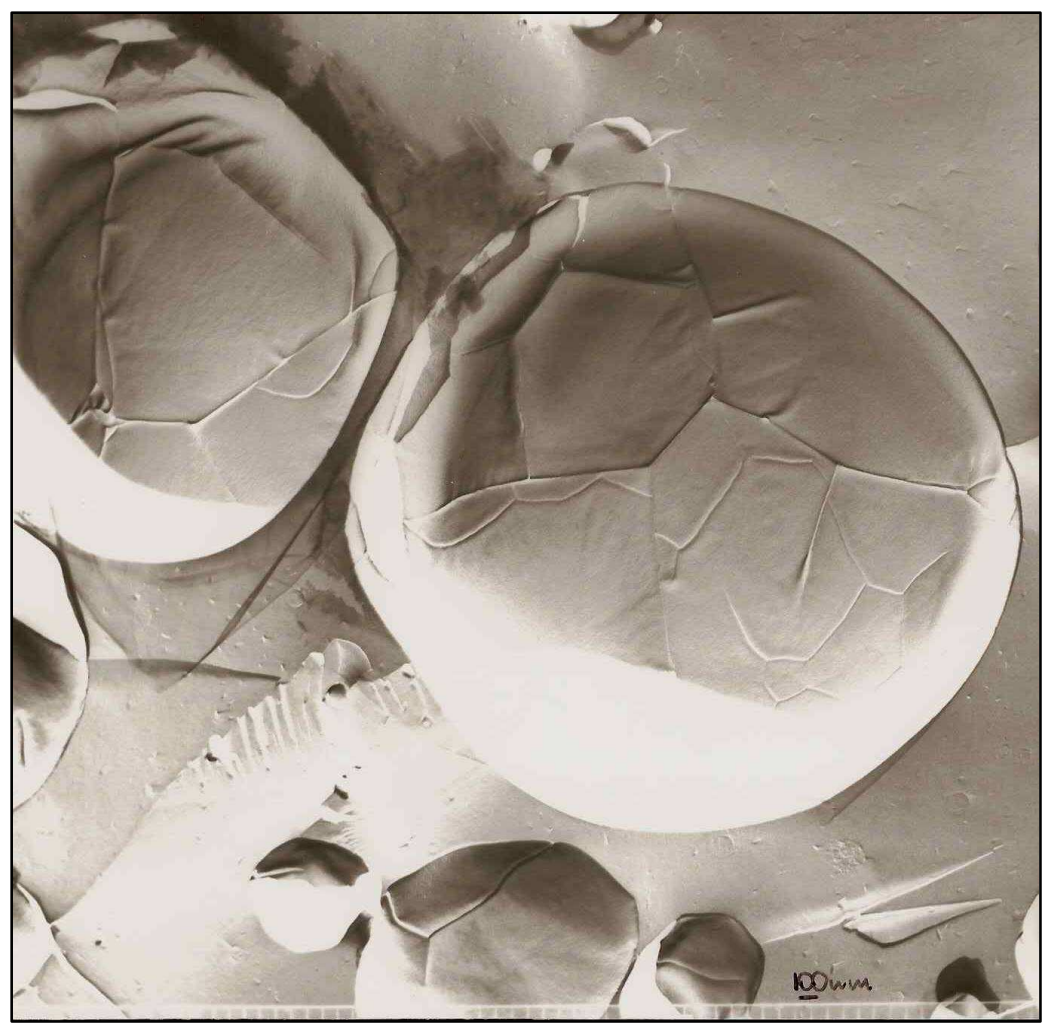

Fig. 1: Lipid-stabilized microbubbles

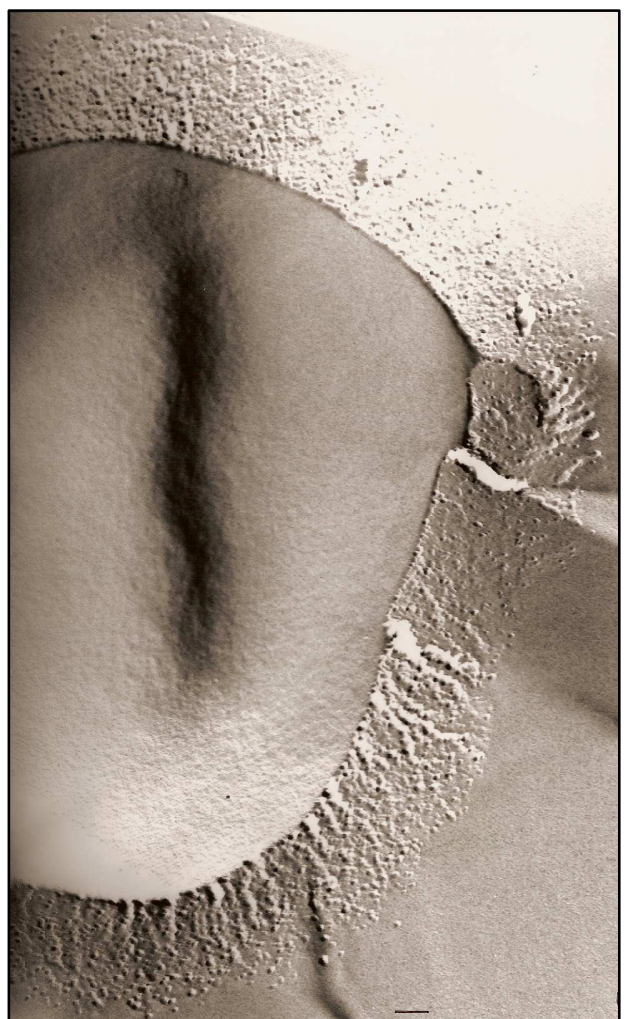

Fig. 3: Polymer-stabilized gas bubble

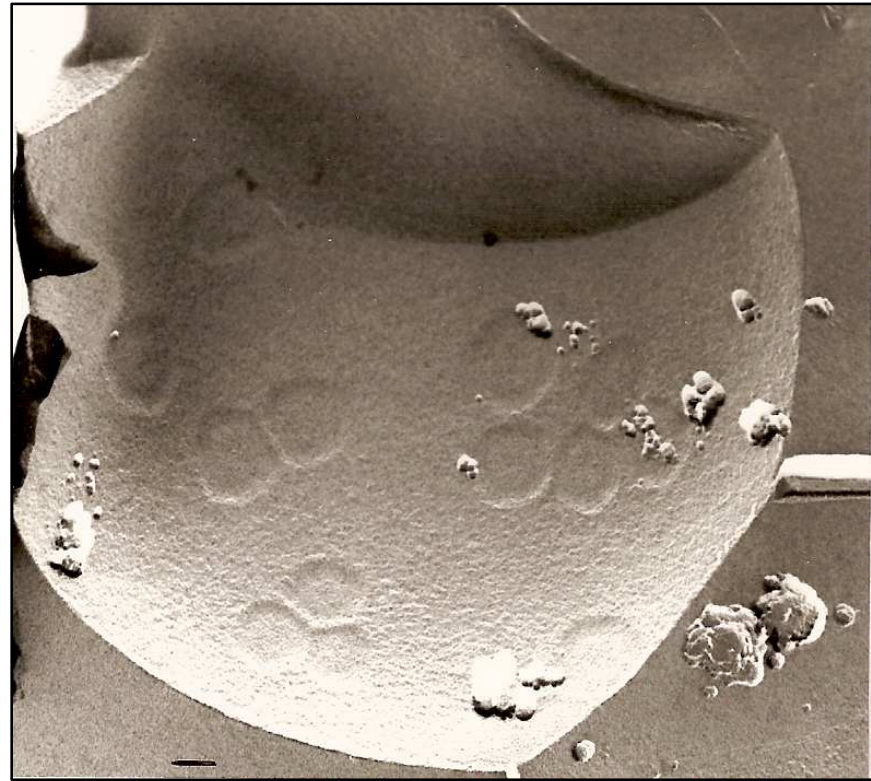

Fig. 2: Domain formation in lipid monolayer

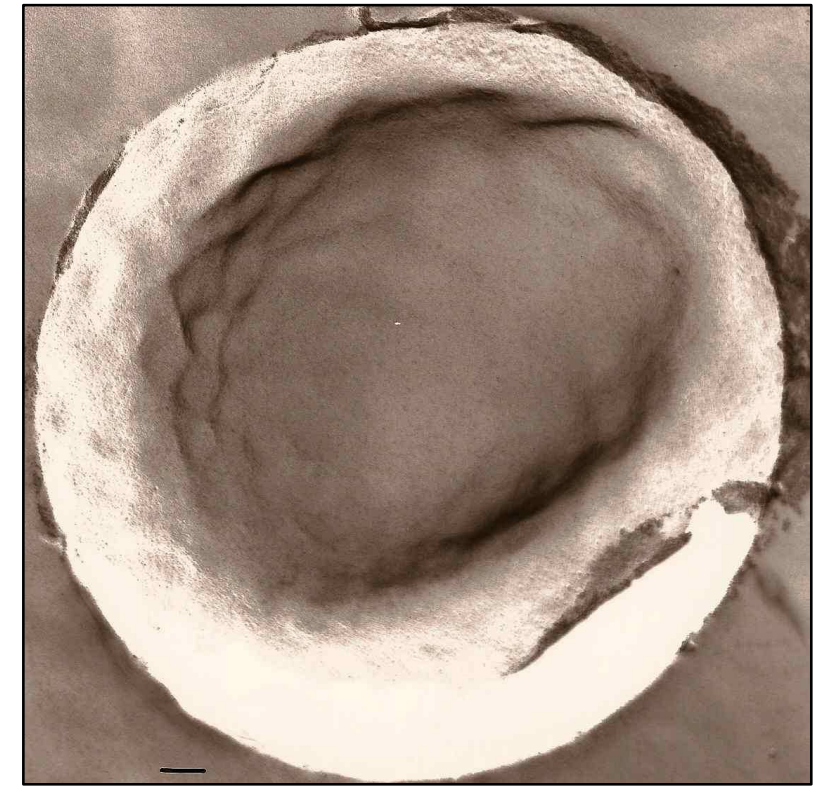

Fig. 4: Lung surfactant microbubble

On all freeze-fracture electron micrographs the bar represents $100 \mathrm{~nm}$ and the shadow direction is running from bottom to top. 